\title{
The subharmonic amplitude of SonoVue increases with hydrostatic pressure at low incident acoustic pressures
}

\author{
Amanda Q.X. Nio*, Alessandro Faraci*, Kirsten Christensen-Jeffries*, Robert J. Eckersley*, \\ Mark J. Monaghan ${ }^{\dagger}$, Jason L. Raymond ${ }^{\ddagger}$, Flemming Forsberg ${ }^{\S}$, Pablo Lamata* \\ *School of Biomedical Engineering and Imaging Sciences, King's College London, United Kingdom; \\ $\dagger$ Department of Cardiology, King's College Hospital, United Kingdom; \\ ${ }^{\ddagger}$ Department of Engineering Science, University of Oxford, United Kingdom;

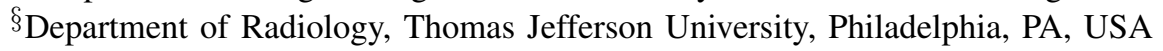 \\ Email: nio@aqxn.info
}

\begin{abstract}
Physiologically important pressures in the heart and aorta are currently assessed with invasive pressure catheters. The subharmonic signal from microbubble ultrasound contrast agents, however, may be exploited to estimate pressures noninvasively. The objective of this work was (i) to develop a static phantom from commercially-available components for easy replication across different laboratories, and (ii) to investigate the subharmonic response of the ultrasound contrast agent SonoVue (Bracco Spa, Milan, Italy) at physiological pressures within this phantom. A phantom capable of maintaining 0-200 $\mathrm{mmHg}$ static pressures was developed using a cell culture cassette with Luer connections. SonoVue was added $(0.4 \mu \mathrm{L} / \mathrm{mL})$ and radiofrequency data were recorded on the ULtrasound Advanced Open Platform (ULA-OP) from 3.5-100\% scanner acoustic output levels (transmit frequency $5 \mathrm{MHz}$, 16-cycle pulse, pulse-inversion). Signal processing was performed to extract the mean subharmonic amplitude in a $1 \mathrm{MHz}$ bandwidth (2-3 MHz). A single growth phase between 75-325 kPa peak-to-peak acoustic pressures was observed at ambient pressure $(0 \mathrm{mmHg})$. Within this growth phase, SonoVue exhibited an increase in subharmonic amplitude from 0-75 $\mathrm{mmHg}$ hydrostatic pressure, a plateau between 75 $125 \mathrm{mmHg}$, and a decrease from $125-200 \mathrm{mmHg}$. The maximum sensitivity of SonoVue to hydrostatic pressure up to $75 \mathrm{mmHg}$ was observed at $152 \mathrm{kPa}$ peak-to-peak acoustic pressure $(0.13$ $\left.\mathrm{dB} / \mathrm{mmHg}, r^{2}=0.99\right)$. This increase in subharmonic signal could have arisen from compression-only behavior as predicted by the Marmottant model, and may have clinical applications in estimating diastolic pressures non-invasively.
\end{abstract}

Keywords: ultrasound contrast agents, subharmonic imaging, pulse-inversion, acoustic output, hydrostatic pressure

\section{INTRODUCTION}

Microbubble ultrasound contrast agents are currently used clinically to improve tissue-to-blood delineation in the heart [1], amongst other applications. The enhanced signal generated by ultrasound contrast agents is due to differences in acoustic impedance and compressibility between microbubbles and

This work was supported in part by a Sir Henry Dale Fellowship funded jointly by the Wellcome Trust and the Royal Society (099973/Z/12/Z; PL), and grants from the BHF Centre of Excellence at King's College of London (RE/13/2/30182), and Action Medical Research jointly with Great Ormond Street Hospital Children's Charity (GN2401). Commercially-available contrast agents were provided by Bracco Spa (SonoVue). their surrounding media [2]. Motivated by the non-invasive nature and low cost of using ultrasound contrast compared with traditional imaging methods, such as magnetic resonance imaging and computerised tomography scans, research is ongoing to develop the novel application of these microbubbles as pressure sensors [3], [4]. Successful implementation of microbubbles as accurate pressure sensors would enable noninvasive estimations of cardiac and large artery pressures [5], beyond the limited and only relative measurements currently available via the modified Bernoulli equation and Doppler imaging in echocardiography.

In the laboratory, ultrasound contrast agents have been found to generate an enhanced subharmonic signal that is linearly and negatively correlated to hydrostatic pressure [3], [6], [7]. The underlying physics leading to this phenomenon are still unclear, but it is likely that changes in microbubble size with hydrostatic pressure, and thus changes in its resonant frequency play a role [6], [8]. This property of ultrasound contrast agents arises when they are insonated with an incident acoustic pressure corresponding to their "growth phase", which can be identified by an increase in subharmonic amplitude with incident acoustic pressure [4]. The phases before and after this growth phase are known as the occurrence and saturation phases, respectively, during which the subharmonic signal is stable despite changes in incident acoustic pressure.

Research into ultrasound contrast agents as pressure sensors has thus far been limited by custom-made pressure chambers, which imposes a barrier on other laboratories seeking to replicate, adopt and extend the seminal work above. Thus, our first objective was to develop a static phantom from commercially-available components for easy replication across different laboratories. Our second objective was to investigate the subharmonic response of the ultrasound contrast agent SonoVue (Bracco Spa, Milan, Italy) at physiological pressures within this phantom. SonoVue is widely used in Europe, but has not yet been established as a potential pressure sensor. We hypothesised that the subharmonic signal of SonoVue would exhibit (i) a growth phase with increasing incident 
acoustic pressures, and (ii) a negative linear relationship with hydrostatic pressure within this growth phase.

\section{MATERiAls AND Methods}

\section{A. Static pressure chamber}

A phantom capable of maintaining 0-200 $\mathrm{mmHg}$ static pressures was developed using a cell culture cassette with Luer connections (CLINIcell 25, $175 \mu \mathrm{m}$ membrane, Mabio International, France), and submerged in a water bath (Fig. 1). A $1.5 \mathrm{~mm}$ magnetic stirrer was inserted into the cassette to maintain a homogenous concentration of microbubbles within the cassette chamber (chamber width $3.7 \mathrm{~mm}$ ). High pressure PVC tubing was secured to the cassette via the two Luer ports (900 PSI, Cole-Parmer, UK), and led out of the water bath to entry and exit Luer stopcocks for administering microbubble solution. Prior to the exit stopcock, a pressure sensor (PRESSS-000 sensor, PendoTech, USA) was connected and positioned outside the water bath at the same height as the middle of the submerged cassette. The pressure sensor was connected to a digital pressure meter (INFCS-112B meter, Newport Electronics, Inc., USA) for real-time chamber pressures. The pressure meter was calibrated from room pressure $(0 \mathrm{mmHg})$ to 147 $\mathrm{mmHg}$ using a $2 \mathrm{~m}$ water column, and the calibration was verified with a $1.5 \mathrm{~m}$ water column $(110.3 \mathrm{mmHg})$. Layers of acoustically-absorbent open-cell foam were positioned behind the cassette to aid identification of the region of interest containing microbubbles - these layers masked the reflections and artifacts created beyond the region of interest (i.e. the chamber inside the cassette).

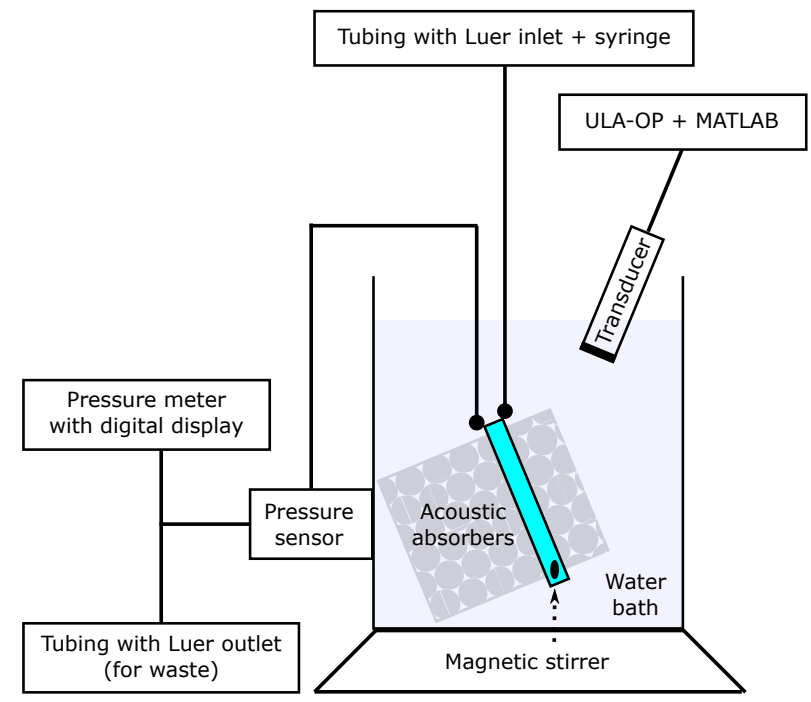

Fig. 1. Schematic representation of the static phantom. The CLINIcell (in blue) was submerged in a water bath with layers of acoustically-absorbent foam in front and behind it. The transducer was positioned at a $45^{\circ}$ angle relative to the window of the CLINIcell. SonoVue was added to the CLINIcell with a syringe, via a Luer stopcock. Luer connections ensured that the CLINIcell chamber was air-tight to maintain stable hydrostatic pressures.

\section{B. Attenuation and insertion loss}

A $12 \mathrm{~mm}$ layer of open-cell melamine foam (Basotect, BASF, Germany) was positioned in front of the cassette to create a layer of attenuation between the ultrasound probe and the microbubbles, to better mimic conditions in vivo. To measure the attenuation resulting from this foam and the insertion loss through one window of the cell culture cassette, a pair of broadband transducers (Panametrics V311, $12.7 \mathrm{~mm}$ diameter, $59 \mathrm{~mm}$ focal length, $10 \mathrm{MHz}$ center frequency; Panametrics V310, $6.35 \mathrm{~mm}$ diameter, $5 \mathrm{MHz}$ center frequency; Olympus NDT, Waltham, MA, USA) were used to acquire the through-transmission spectrum using a broadband substitution technique as previously described [9]. Measurements were conducted in an $8 \mathrm{~L}$ acrylic tank $(45 \times 12 \times 15 \mathrm{~cm})$ filled with distilled water. An ultrasound pulser-receiver (DPR300, JSR Ultrasonics, Pittsford, NY, USA) was used to generate the excitation pulse and amplify the received signal (20-50 dB gain). Received waveforms were averaged (typically 64 traces per acquisition), digitized (LT264, LeCroy, Chestnut Ridge, NY, USA), and transferred to a computer for analysis using MATLAB (The MathWorks, Inc., Natick, MA, USA).

Attenuation through the open-cell melamine foam was estimated to be $1.75 \mathrm{~dB} / \mathrm{cm}$ at transmit frequency $5 \mathrm{MHz}$. Based on a $45^{\circ}$ orientation between the ultrasound transducer and the cell culture cassette, the insertion loss was $3.22 \mathrm{~dB}$ at transmit frequency $5 \mathrm{MHz}$. This orientation increased the effective width of the cassette chamber to $5.2 \mathrm{~mm}$. The total acoustic signal loss through the foam and cassette window in our static phantom configuration was thus estimated as $6.19 \mathrm{~dB}$, and used to estimate the acoustic pressure within the cassette chamber (acoustic pressure in a water bath $\times 10^{-6.19 / 20}$ ).

\section{Experimental protocol}

SonoVue was reconstituted according to manufacturer's instructions and diluted in gas-equilibrated water, based on the concentration used in the clinic $(0.4 \mu \mathrm{L} / \mathrm{mL}$ water $)$. With the exit port open and the magnetic stirrer circulating in the cassette chamber, approximately $25 \mathrm{~mL}$ of diluted microbubble solution was added to the static phantom $(\approx 0.5 \mathrm{~mL} / \mathrm{s}$; cassette chamber volume $10 \mathrm{~mL}$ ). The exit port was then closed and hydrostatic pressure was increased by adding more microbubble solution, with immediate feedback provided by the pressure meter. Radiofrequency data for pulse-inversion were recorded at transmit frequency $5 \mathrm{MHz}$ with a 16-cycle pulse (bandwidth 3-7 MHz, LA332E Marzo 2014, Esaote, Genoa, Italy) on the ULtrasound Advanced Open Platform (ULA-OP, MSD Lab, University of Florence, Italy). The maximum acoustic pressure generated was $1.81 \mathrm{MPa}$, with pulse length $4.2 \mathrm{~mm}$, as measured with a $0.5 \mathrm{~mm}$ hydrophone (SN1832, Precision Acoustics Ltd, Dorchester, UK) in a water bath. This pulse length did not exceed the $5.2 \mathrm{~mm}$ cassette chamber width with a $45^{\circ}$ angle of insonation.

Echo waveforms were first recorded at ambient hydrostatic pressure $(0 \mathrm{mmHg}$ on the pressure meter) from 3.5$100 \%$ scanner acoustic output levels $(27-890 \mathrm{kPa}$ peak-topeak acoustic pressure after correcting for signal loss through the foam and cassette window; $n=40$ ) - to determine the acoustic pressure range that elicited the growth phase response of SonoVue. Subsequently, data were recorded from 
9-42\% scanner acoustic output levels (67-330 kPa peak-topeak acoustic pressure after correcting for signal loss; $n=20$ ), at hydrostatic pressures $200-0 \mathrm{mmHg}$ in $25 \mathrm{mmHg}$ intervals. The microbubble solution in the phantom was replenished after each set of incremental acoustic output levels. Two sets of data were recorded at each hydrostatic pressure level. All experiments were performed at room temperature $\left(\approx 21^{\circ} \mathrm{C}\right)$, and were completed within $3.5 \mathrm{~h}$ of microbubble reconstitution.

\section{Extracting and analyzing the subharmonic signal}

The mean signal amplitude in a $1 \mathrm{MHz}$ bandwidth around the theoretical subharmonic frequency (i.e. from $2-3 \mathrm{MHz}$; Fig. 2A) was extracted offline using MATLAB. A Butterworth infinite impulse response (IIR) band-pass filter was implemented to isolate the signal within the subharmonic bandwidth. Based on the greyscale B-mode image during data acquisition, a $15.5 \times 3 \mathrm{~mm}$ region of interest was defined and adjusted for the signal shift due to filtering (Fig. 2B). The average subharmonic amplitude was calculated as the mean amplitude across three frames of the region of interest (i.e. $3 \times 64$ lines/frame).
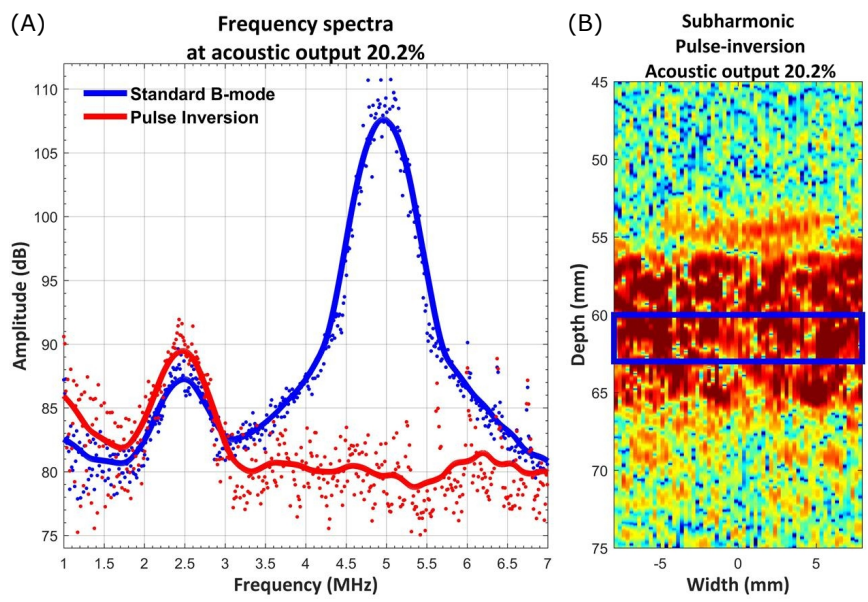

Fig. 2. (A) Smoothed frequency spectra illustrating the enhanced subharmonic signal, and cancellation of the fundamental signal with pulse-inversion compared with standard B-mode. Individual points represent the mean across 64 lines in one full frame. (B) Recreated 2D image of the subharmonic amplitude, with the rectangular region of interest defined by a blue border. In this example, data were recorded at $75 \mathrm{mmHg}$ and $152 \mathrm{kPa}$ peak-to-peak acoustic pressure (scanner acoustic output level 20.2\%).

Linear regressions between subharmonic amplitude and hydrostatic pressure $(0-75 \mathrm{mmHg}$ and $125-200 \mathrm{mmHg}$, separately) were applied at each scanner acoustic output level to identify the maximum sensitivity of SonoVue to changes in hydrostatic pressure.

\section{RESUlTS AND DISCUSSION}

A. Subharmonic amplitude of SonoVue across the full range of scanner acoustic output levels at ambient pressure

SonoVue generated subharmonic signals that followed the characteristic occurrence, growth and saturation phases previously observed in microbubble ultrasound contrast agents [4]
(Fig. 3). The occurrence phase for SonoVue ranged from $27-$ $75 \mathrm{kPa}$ peak-to-peak acoustic pressures (corrected for signal loss through the foam and cassette window). The growth phase for SonoVue extended from 75-325 kPa peak-to-peak acoustic pressures, characterised by substantial increases in mean subharmonic amplitude with increasing incident acoustic pressure. Within this growth phase, the subharmonic amplitude of microbubbles has been suggested to vary with hydrostatic pressure [4]. This range of incident acoustic pressures was thus demarcated and applied in the following experiments at $200-0 \mathrm{mmHg}$ hydrostatic pressures. Above $325 \mathrm{kPa}$ peak-topeak acoustic pressures and up to the maximum capacity of the hardware in this study $(890 \mathrm{kPa})$, the subharmonic signal from SonoVue plateaued at its saturation level.

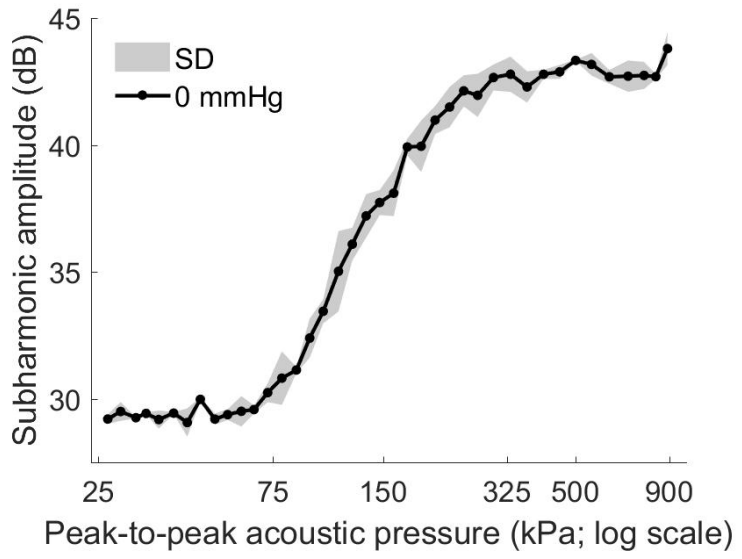

Fig. 3. Mean subharmonic amplitude of SonoVue at ambient hydrostatic pressure $(0 \mathrm{mmHg})$ across the full range of scanner acoustic pressures at transmit frequency $5 \mathrm{MHz}$ and a 16-cycle pulse with pulse-inversion $(n=2)$. Horizontal axis: water bath acoustic pressures corrected for $6.19 \mathrm{~dB}$ signal loss through the melamine foam and cassette window. SD: 1 standard deviation.

B. Subharmonic amplitude of SonoVue exhibits an inverse Vshaped behavior from 0-200 $\mathrm{mmHg}$ hydrostatic pressure

We observed an increase in the subharmonic signal of SonoVue from $0-75 \mathrm{mmHg}$ hydrostatic pressure, a plateau in the signal between $75-125 \mathrm{mmHg}$, and then a decrease from $125-200 \mathrm{mmHg}$ (Fig. 4). This behavior is in contrast to our hypothesis, which was based on previous work demonstrating a linear decrease in the subharmonic signal of the ultrasound contrast agents Sonazoid, Optison, Levovist and Definity with increasing hydrostatic pressure up to $186 \mathrm{mmHg}$ [7]. We speculate that the increase and plateau in subharmonic signal observed in this study could have arisen from compressiononly behavior of SonoVue microbubbles [6]. This behavior refers to asymmetric microbubble oscillations involving mainly compression, instead of expansion. Frinking and colleagues have previously shown an increase in subharmonic signal with experimental perfluorobutane $\left(\mathrm{C}_{4} \mathrm{~F}_{10}\right)$ phospholipidshell microbubbles at $100 \mathrm{kPa}$ peak-to-peak acoustic pressure (50 kPa peak-negative acoustic pressure and assuming a symmetrical pulse), and additionally, attributed their empirical findings to compression-only behavior with simulations using 
the Marmottant model [6], [10]. However, our results do not fully match Frinking's findings, as we observed a subsequent decrease in the subharmonic signal from 125-200 mmHg. This discrepancy could be due to microbubble properties specific to SonoVue, and further experimental and computational studies are necessary to discern the underlying mechanisms.
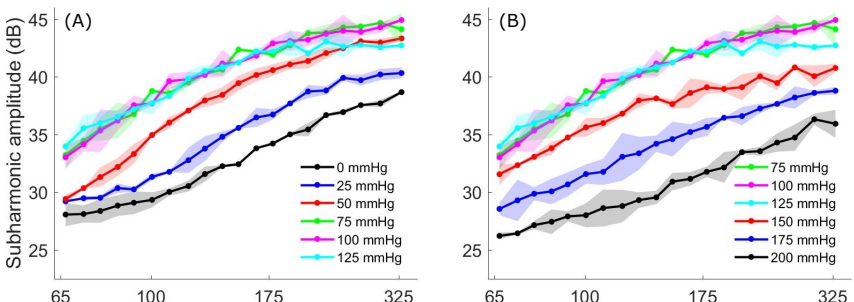

Peak-to-peak acoustic pressure (kPa; log scale) Peak-to-peak acoustic pressure (kPa; log scale)

Fig. 4. Mean subharmonic amplitude of SonoVue at (A) $0-125 \mathrm{mmHg}$ and (B) $75-200 \mathrm{mmHg}$ hydrostatic pressure. Data at $75-125 \mathrm{mmHg}$ are identical in (A) and (B) for reference. Translucent shading indicates 1 standard deviation around the mean $(n=2)$. Horizontal axis: water bath acoustic pressures corrected for $6.19 \mathrm{~dB}$ signal loss through the melamine foam and cassette window; scanner acoustic output levels 9-42\%.

The subharmonic response of SonoVue to changes in hydrostatic pressure could potentially be applied to estimate pressures in vivo. For example, the linear increase in subharmonic signal from $0-75 \mathrm{mmHg}$ could be used to estimate diastolic pressures in the heart, which are critical for the assessment of the diastolic performance [5]. In addition, the linear decrease in subharmonic signal from $125-200 \mathrm{mmHg}$ could be applied to detect elevated aortic pressures. In this study, the maximum sensitivity of SonoVue to $0-75 \mathrm{mmHg}$ hydrostatic pressures was $0.13 \mathrm{~dB} / \mathrm{mmHg}\left(r^{2}=0.99\right.$; Fig. 5$)$ at $152 \mathrm{kPa}$ peak-topeak acoustic pressure (scanner acoustic output level 20.2\%; $309 \mathrm{kPa}$ before correcting for signal loss). This pressure sensitivity is more than $1.5 \times$ that previously reported by Halldorsdottir and colleagues for the ultrasound contrast agents Sonazoid $(0.08 \mathrm{~dB} / \mathrm{mmHg})$, Definity $(0.07 \mathrm{~dB} / \mathrm{mmHg})$ and Optison $(0.06 \mathrm{~dB} / \mathrm{mmHg})$ [7].

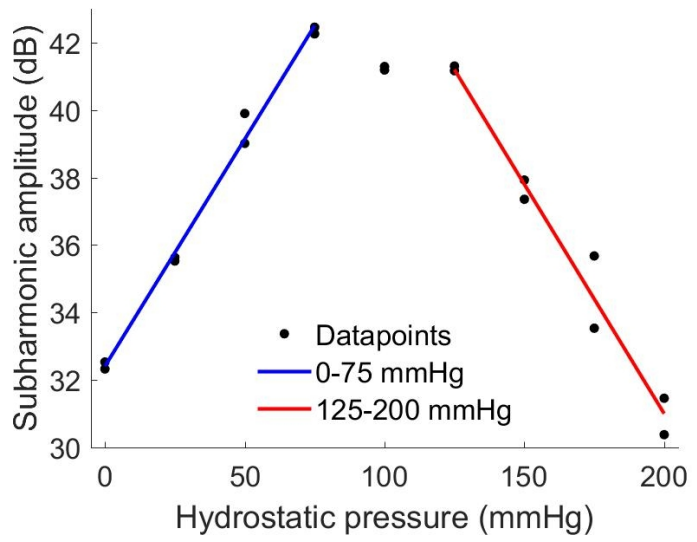

Fig. 5. Subharmonic amplitude of SonoVue from 0-200 $\mathrm{mmHg}$ hydrostatic pressure at $152 \mathrm{kPa}$ peak-to-peak acoustic pressure $(n=2)$, with maximum sensitivity to $0-75 \mathrm{mmHg}$ hydrostatic pressure $\left(0.13 \mathrm{~dB} / \mathrm{mmHg} ; r^{2}=0.99\right)$. Sensitivity to $125-200 \mathrm{mmHg}$ at this acoustic pressure was $-0.15 \mathrm{~dB} / \mathrm{mmHg}$ $\left(r^{2}=0.97\right)$.

\section{Limitations, implications and future work}

This work builds upon and extends previous research on applying ultrasound contrast agents to estimate pressure. Future work investigating SonoVue as a potential pressure sensor should include different transmit frequencies, particularly $<5 \mathrm{MHz}$ for cardiac applications. Shorter pulse-lengths may be examined to improve spatial resolution, but this would reduce the resolution of the subharmonic peak, and thus a considered trade-off is required. In addition, assessing the subharmonic signal through varying depths of acousticallyabsorbent foam and in a dynamic flow phantom will provide insight into the robustness of SonoVue as a pressure sensor in the human body.

\section{CONCLUSION}

In conclusion, we have developed a static phantom capable of maintaining physiological pressures, and have used this to investigate the subharmonic signal of SonoVue at 0$200 \mathrm{mmHg}$ hydrostatic pressure. Surprisingly, SonoVue exhibited an increase in subharmonic amplitude from $0-75 \mathrm{mmHg}$ hydrostatic pressure, a plateau between $75-125 \mathrm{mmHg}$, and a decrease from 125-200 mmHg. This behavior of SonoVue in response to hydrostatic pressure could potentially be applied to estimate in vivo pressures non-invasively.

\section{REFERENCES}

[1] S. L. Mulvagh, H. Rakowski, M. A. Vannan et al., "American Society of Echocardiography consensus statement on the clinical applications of ultrasonic contrast agents in echocardiography," J Am Soc Echocardiogr vol. 21, no. 11, pp. 1179-1201, 2008.

[2] J. R. Eisenbrey, A. Sridharan, J.-B. Liu, and F. Forsberg, "Recent experiences and advances in contrast-enhanced subharmonic ultrasound," Biomed Res Int, vol. 2015, Article ID 640397, 6 pages, 2015.

[3] K. S. Andersen and J. A. Jensen, "Impact of acoustic pressure on ambient pressure estimation using ultrasound contrast agent," Ultrasonics, vol. 50, no. 2, pp. 294-299, 2010.

[4] J. K. Dave, V. G. Halldorsdottir, J. R. Eisenbrey et al., "On the implementation of an automated acoustic output optimization algorithm for subharmonic aided pressure estimation," Ultrasonics, vol. 53, no. 4, pp. 880-888, 2013.

[5] J. Xi, W. Shi, D. Rueckert, R. Razavi, N. P. Smith, and P. Lamata, "Understanding the need of ventricular pressure for the estimation of diastolic biomarkers," Biomech Model Mechanobiol, vol. 13, pp. 747757, 2013

[6] P. J. Frinking, E. Gaud, J. Brochot, and M. Arditi, "Subharmonic scattering of phospholipid-shell microbubbles at low acoustic pressure amplitudes," IEEE Trans Ultrason Ferroelectr Freq Control, vol. 57, no. 8, pp. 1762-1771, 2010.

[7] V. G. Halldorsdottir, J. K. Dave, L. M. Leodore, J. R. Eisenbrey, S. Park, A. L. Hall, K. Thomenius, and F. Forsberg, "Subharmonic contrast microbubble signals for noninvasive pressure estimation under static and dynamic flow conditions," Ultrason Imaging, vol. 33, no. 3, pp. 153164,2011

[8] A. Katiyar, K. Sarkar, and F. Forsberg, "Modeling subharmonic response from contrast microbubbles as a function of ambient static pressure," $J$ Acoust Soc Am, vol. 129, no. 4, pp. 2325-2335, 2011.

[9] J. L. Raymond, K. J. Haworth, K. B. Bader, K. Radhakrishnan, J. K. Griffin, S.-L. Huang, D. D. McPherson, and C. K. Holland, "Broadband attenuation measurements of phospholipid-shelled ultrasound contrast agents," Ultrasound Med Biol, vol. 40, no. 2, pp. 410-421, 2014.

[10] P. Marmottant, S. van der Meer, M. Emmer, M. Versluis, N. de Jong, S. Hilgenfeldt, and D. Lohse, "A model for large amplitude oscillations of coated bubbles accounting for buckling and rupture," J Acoust Soc Am, vol. 118, no. 6, pp. 3499-3505, 2005. 